\title{
Possible Origin of Clusters in Ultra-High-Energy Cosmic Rays
}

\author{
A.V. Uryson \\ Lebedev Physics Institute, 117924, Moscow, Russia
}

E-mail: uryson@sci.lebedev.ru

\begin{abstract}
We estimate the detection rate of ultrahigh-energy cosmic rays on ground-based arrays by assuming that the cosmic-ray sources are active galactic nuclei. We analyze the cases of detection of clusters, several particles that arrived, within the error limits, from the same area of the sky. The adopted model is shown to explain the detection rate of clusters on the AGASA array.
\end{abstract}

\section{Introduction}

The origin of ultra-high-energy cosmic rays (UHECRs), $E>4 \times 10^{19} \mathrm{eV}$, has not yet been elucidated. In our opinion, the sources of UHECRs are active galactic nuclei (AGNs). This is an old hypothesis that was discussed in the late 1960s and with which the AGN space density estimates [1](Berezinskii et al. 1990) were consistent. The identification of CR sources performed in [2-6] has shown that Seyfert nuclei with redshifts $z<0.01$ and Blue Lacertae objects (BL Lac) are possible sources of UHECRs.

On their way from the source to the Earth, UHECRs are deflected by magnetic fields in the intergalactic space and in the Galaxy. When identifying the sources, we assumed that CRs are deflected by the intergalactic magnetic fields through no more than $9^{0}$. The intergalactic magnetic fields are $B<10^{-9} \mathrm{G}[7]$, and this condition is satisfied for the particles arrived from distances of $\sim 50 \mathrm{Mpc}$, i.e., emitted by Seyfert nuclei with $z<0.01$ [8]. BL Lac objects are hundreds of megaparsecs away, but even for such distant sources, the deflections may not exceed a few degrees if the filamentary structure of the intergalactic magnetic fields is taken into account [9]. The field in the Galaxy has regular and irregular components; the field strength is $B \sim 10^{-6} \mathrm{G}$. In the disk, the field is regular in the spiral arms and directed along them; in the halo, the regular component is perpendicular to the disk [7]. The deflections of particles in the regular field depend on the particle arrival direction and can be negligible. In the irregular field, the deflections do not exceed $1^{0}$. Therefore, our identification is valid for CRs arrived from fairly high Galactic latitudes (in all our identification works, we selected UHECRs depending on the Galactic latitude of their arrival).

Our identification of sources was based on a statistical analysis. The major CR sources can be revealed in this way, but it is dificult to exclude other hypotheses. For instance, there are UHECR particles that arrived from areas of the sky where there are neither Seyfert nuclei with $z<0.01$ nor BL Lac objects. This may be because the catalogs of objects are incomplete; they do not contain all objects of a given type, and, as a result, some particles arrive from "empty" areas of the sky. (For this reason, the sources can be identified only statistically.) However, a different explanation is possible: the statistical analysis has revealed the major CR sources, but there are also other, less efficient or rarer sources. CRs in the arrival direction of which no object of the "major" type is observed originate from these "minor" sources. To reliably establish the CR sources, we must find out whether conditions for UHECR acceleration exist in the identified objects and compare the model predictions with experimental data. 
The particle acceleration in the identified objects was considered in $[8,10-12]$. It was shown that conditions for particle acceleration to energies of $10^{21} \mathrm{eV}$ exist in the identified objects. The CR spectra near the Earth obtained in the model under consideration agree, within the error limits, with the measured spectra [13].

Here, we analyze the clusters detected in UHECRs, groups of particles with coincident (within the error limits) arrival directions, and consider how the existence of clusters can be explained in our model.

\section{The UHECR detection rate from a single source}

Let us estimate the detection rate of UHECRs emitted by a single source on ground-based arrays with an area $S \approx 10 \mathrm{~km}^{2}$ (the Yakutsk, Haverah Park, Akeno, and Volcano Ranch arrays have approximately the same area) and $S \approx 100,3000 \mathrm{~km}^{2}$ (the areas of the AGASA and Pierre Auger arrays).

Let us first consider Seyfert nuclei. To provide the observed intensity of CRs with energies $E>5 \times 10^{19} \mathrm{eV}, I(E) \approx 10^{39}-10^{40}\left(\mathrm{~cm}^{2} \mathrm{~s} \mathrm{sr} \mathrm{eV}\right)^{-1}$ [14], a Seyfert nucleus spends an estimated power of $L_{S} \approx 10^{39}-10^{40} \mathrm{erg} \mathrm{s}^{-1}$ on the emission of UHECRs [4,8].

This estimate was obtained by assuming that the particles are emitted by the source isotropically. However, in our acceleration model $[8,12]$, the particles are accelerated in a jet and are emitted in a directed beam with an opening half-angle of $\theta \leq 6.5 \times 10^{-4}$. Taking into account the possible conversion of the accelerated particles from the charged into the neutral state and back [15], we find that UHECRs are emitted in a cone with an angle of $20^{\circ}$. It thus follows that the sources of the detected CRs are Seyfert nuclei oriented in such a way that the angle $i$ between the line of sight and the normal to the plane does not exceed $10^{0}$. In the catalog [16], the fraction of such nuclei (among those with redshifts $z<0.01$ ) is $\sim 0.15$. However, the Seyfert nuclei identified as possible CR sources are oriented differently; the mean angle $i$ is $\approx 52^{0}$. If these objects are actually CR sources, then they emit uncollimated beams of particles. UHECR particles can be emitted in an uncollimated way from an accretion disk in the model [17]. We adopt this model by assuming that CRs emerging from the source in a cone with an angle $\theta \approx 50^{\circ}$ are detected on Earth.

At a given CR intensity near the Earth, the power of a source with emission in a solid angle $\Omega_{S}$ is a factor of $\Omega_{S} /(4 \pi)$ lower than that of an isotropic source. For an angle $\theta \approx$ $50^{0}$, the solid angle is $\Omega_{S} \approx 2$, and the UHECR beam power is $L_{S}^{b} \approx 2 \times 10^{39} \mathrm{erg} \mathrm{s}^{-1}$ if $I(E) \approx 10^{-39}\left(\mathrm{~cm}^{2} \mathrm{~s} \mathrm{sr} \mathrm{eV}\right)^{-1}$ and $L_{S} \approx 10^{40} \mathrm{erg} \mathrm{s}^{-1}$. Assuming that the energy spectrum of the accelerated particles is a power law [4] and, therefore, the overwhelming majority of particles has an energy $E_{0} \approx 5 \times 10^{19} \mathrm{eV}$, we find that the source emits $N_{S}=L_{S}^{b} / E_{0} \approx$ $2 \times 10^{31}$ (particle $\mathrm{s}^{-1}$ ). At distance $R$ from the source, the beam particles cross the area $\pi R^{2} \Omega_{S}$, and the particle flux (the number of particles crossing a unit area perpendicular to the beam axis per unit time) is $N=L_{S}^{b} /\left(R^{2} \Omega_{S} E_{0}\right)$ (this relationship is formally identical to the expression for the number of particles in a unit solid angle for isotropic emission $\left.N=L_{S} /\left(4 \pi R^{2} E_{0}\right)\right)$.

Let the source be at a distance of $R=16 \mathrm{Mpc}$. This is the distance at which the maximum in the spatial distribution of Seyfert nuclei with redshifts $z<0.01$ from the catalog [18] is located for the Hubble constant $H=75 \mathrm{~km} \mathrm{Mpc}^{-1} \mathrm{~s}^{-1}$. On Earth, the particle flux from this source is $N \approx 4.3 \times 10^{-21}\left(\mathrm{~cm}^{2} \mathrm{~s}\right)^{-1}$, and arrays with areas $S \approx 10,100$, and $3000 \mathrm{~km}^{2}$ detect $0.012,0.12$, and $\sim 4$ particles per year, respectively. Hence, we find that no doublets of particles are detected from a single source on arrays with $S \sim 10 \mathrm{~km}^{2}$. On the AGASA array $\left(S \approx 100 \mathrm{~km}^{2}\right)$, a doublet of particles from a single source can be detected in an observing time of $T>10 \mathrm{yr}$. On the Pierre Auger array $\left(S \sim 3000 \mathrm{~km}^{2}\right)$, a cluster from a single source can be detected during a year of operation. In the section "Discussion" we list the reasons why these estimates can be considerably lower.

Let us now determine the detection rate if the UHECR sources are BL Lac objects. In 
these objects, the jets are directed toward the observer (here, we do not touch on uniformity or nonuniformity of the $\mathrm{CR}$ intensity distribution in different regions of the intergalactic space). Consider the possible values of the parameters that are necessary for our estimates by assuming that the particles are accelerated in the source in accordance with the model [10].

In the model [10], the power spent on UHECR emission is $L_{B L}=1.5 \times 10^{12} L_{\odot}$, where $L_{\odot}$ is the luminosity of the Sun. At $L \approx 3.86 \times 10^{33} \mathrm{erg} \mathrm{s}^{-1}$, we have $L_{B L} \approx 6 \times 10^{45} \mathrm{erg} \mathrm{s}^{-1}$. Since the particle in this model are accelerated by an electric field, we assume the original CR spectrum to be monoenergetic. The energy of the accelerated protons in the model reaches $\sim 10^{20}-10^{21} \mathrm{eV}[10,11]$, in agreement with the maximum value of $10^{21} \mathrm{eV}$ in the sources obtained in [19, 20] and with our estimates [13]. According to our estimates obtained by comparing the calculated and measured UHECR spectra [13], the CR emission power is $L_{B L} \sim 10^{43}-10^{44} \mathrm{erg} \mathrm{s}^{-1}$ (this quantity cannot be determined with a better accuracy because of the large measurement errors). The particles are emitted in a directed beam with an opening half-angle $\alpha \approx 7 \times 10^{-7}[10]$.

The fraction of the particles that reached the array with energy $E \geq 4 \times 10^{19} \mathrm{eV}$ depends on the distance of the source due to the CR energy losses in the interactions with the background radiations in the intergalactic space [21, 22]. In what follows, we assume that this fraction is $0.3,0.5,0.7$, and 1 for distances $R \approx 800,700,600 \mathrm{Mpc}$, and $R<600 \mathrm{Mpc}$, respectively. We will disregard the contribution from the sources at $R>800 \mathrm{Mpc}$ (because of the low particle density, the contribution from these sources to the detected flux is an order of magnitude lower than the contribution from the sources at $R<600 \mathrm{Mpc}$ even without including the CR energy losses in the intergalactic space).

Assuming that the source has a redshift $z \approx 0.2$ (this is the redshift at which the maximum in the spatial distribution of BL Lac objects from the catalog [18] is located), i.e., is $R \approx 600$ Mpc away, we estimate the CR detection rate for the following sets of parameters.

(1) Assuming that $L_{B L} \approx 6 \times 10^{45} \mathrm{erg} \mathrm{s}^{-1}$ is the power of the directed beam, we find for $E_{0}=10^{21} \mathrm{eV}$ that an array with a relatively small area, $S \approx 10 \mathrm{~km}^{2}$, detects $\sim 10^{8}$ particles per year from a single source, in conflict with the measurements.

(2) For an isotropic CR emission power $L_{B L} \approx 6 \times 10^{45} \mathrm{erg} \mathrm{s}^{-1}$ (the equivalent power of the directed beam is $L_{B L}^{b} \approx 10^{33} \mathrm{erg} \mathrm{s}^{-1}$ ) and a particle energy of $E_{0}=10^{21} \mathrm{eV}$, an array with an area $S \approx 100 \mathrm{~km}^{2}$ will detect 1.8 particles per year from a single source and a cluster of three or more particles in several years. On an array with $S \approx 10 \mathrm{~km}^{2}$, a doublet of particles can be detected in a time $T>10 \mathrm{yr}$.

(3) Finally, let us consider a set of parameters with an isotropic CR emission power of $L_{B L} \sim 10^{44} \mathrm{erg} \mathrm{s}^{-1}$ (the equivalent power of the directed beam is $L_{B L}^{b} \approx 1.6 \times 10^{31} \mathrm{erg} \mathrm{s}^{-1}$ ) and a particle energy of $E_{0}=10^{21} \mathrm{eV}$. In this case, arrays with an area of 10, 100, and $3000 \mathrm{~km}^{2}$ will detect $5 \times 10^{-4}, 5 \times 10^{-3}$, and 0.15 particles per year, respectively, from a single source. In this model, at $L_{B L} \leq 10^{44} \mathrm{erg} \mathrm{s}^{-1}$, CR clusters from a single source can be detected only on an array with an area $S \sim 3000 \mathrm{~km}^{2}$ in a time $T>10 \mathrm{yr}$.

Below, we analyze the second and third sets of parameters and do not consider the first set, because it is in conflict with the experimental data. Let us turn to the AGASA data.

\section{Analysis of the clusters detected on the AGASA array}

UHECR particles whose arrival directions coincided within a single error, a total of five doublets and one triplet of 63 UHE particles, were detected on the AGASA array over 10 years [23]. A particle detected on the Yakutsk array [24] also falls into the triplet particle region. The detection rate of particles in clusters is $\sim 1-1.5$ particles per year; in doublet C4 (according to the numbering of clusters from [23]), the particles were detected with an interval of almost 10 years.

The UHECR particles most likely arrive from areas of the sky with an enhanced AGN 
density [3]. (The sources in the model with the second set of parameters, as shown above, constitute an exception.) If this is the case, then the particles that form a cluster also arrive from such areas. The size of these areas in equatorial coordinates is $\left(\Delta \alpha \leq 9^{0}, \Delta \delta \leq 9^{0}\right)$. If we assume that the errors in the right ascension and declination are approximately $3^{0}$, then this is the region of a triple error in the determination of the particle arrival direction.

Let us test this assumption. Let us consider the AGNs near the arrival direction of the particles in each cluster and estimate the CR detection rate. The size of the neighborhood in which we will analyze the AGNs is $\left(\Delta \alpha \leq 9^{0}, \Delta \delta \leq 9^{0}\right)$.

Seyfert nuclei with $z<0.01$ fall into the search region of four clusters: C2, C3, C5, and C6. The search region of triplet C2 contains five nuclei from the catalog [18], eight objects from [16], and ten nuclei from the catalog [25]. One object from the catalog [25] falls into the region of doublet $\mathrm{C} 3$ and one object from $[16,18,25]$ falls into the region of cluster C5. There are five, three, and four nuclei from the catalogs $[16,18,25]$, respectively, in the region of doublet C6.

The UHECR flux from the Seyfert nuclei in the region of triplet C2 detected by an array with an area $S \approx 100 \mathrm{~km}^{2}$ is $\sim 0.6$ particles per year, the flux in the region of doublets C3 and C5 is $\sim 0.03$ particles per year, and the flux from the nuclei in the region of cluster C6 is $\sim 2$ particles per year, as estimated from the data of the catalogs [16] or [25].

The fluxes of $\sim 0.03$ particles per year are too low to explain the doublets of particles; the fluxes of $\sim 1$ particles per year are enough for clusters to be detected on the AGASA array. Thus, the model with Seyfert nuclei explains the origin of two clusters.

BL Lac objects fall into the search regions of all the clusters, except doublet C4. Nearby Seyfert nuclei do not fall into the $\mathrm{C} 4$ search region either. This may be attributable to the lowGalactic latitude of the particle arrival, $b \approx-10^{0}$, since this latitude corresponds to the zone of avoidance of galaxies in which relatively fewobjects are observed. The numbers of objects from the catalogs $[16,18]$ that fall into the search regions are respectively 4 and 3 for doublet C1, 9 and 2 for triplet C2, 8 and 4 for cluster C3, 12 and 6 for doublet C5, 13 and 8 for cluster C6.

Let us determine the UHECR flux from the BL Lac objects in the cluster region initially for the third set of parameters. (In our estimates, we disregarded the BL Lac objects with unknown redshifts.) An array with an area $S \approx 100 \mathrm{~km}^{2}$ will detect the following fluxes: $\sim 0.3, \sim 0.4, \sim 0.1, \sim 0.6$, and $\sim 0.45$ particles per year in the regions of clusters $\mathrm{C} 1, \mathrm{C} 2, \mathrm{C} 3$, C5, and C6, respectively. The fluxes $\sim 0.3-0.6$ particles per year are enough to explain the detected clusters of particles.

The fluxes calculated with the second set of parameters will be a factor of $\sim 50$ higher. In this model, the clusters of particles are emitted by single sources.

\section{Discussion}

The above fluxes may have been overestimated. The reasons are the following.

First, the fluxes were obtained by assuming that the array detects the emission from the sources during the entire period of its operation. In fact, the source position in the sky can depend on the time of the day and on the season. As a result, the actual time during which the source emission is detected by the array can be considerably shorter. For instance, if the sky area from which the cluster particles arrive falls into the array survey region $\sim 1 / 2$ day during $\sim 1 / 2$ year, then the detected CR flux from the sources will be a factor of 4 lower than the estimates obtained.

Second, the arrays usually select showers with polar angles of the arrival direction $\Theta<$ $30^{0}-45^{0}$; therefore, no more than half of the emission from a given source is detected. As a result, the estimated $\mathrm{CR}$ detection rate may be a factor of $\sim 2-10$ higher than the measured values. For these reasons, although the second set of parameters in the BL Lac model yields a high CR flux, it is probably suitable for explaining the detected clusters. 
In addition, the activity of BL Lac objects can be not constant, but quasi-periodic, with a period of 4 to $\sim 25 \mathrm{yr}$ with a relatively short duration of the active state [26]. If this is the case, then the clusters can be formed through the emission and the subsequent "turn-off" of a single source in the model with the second set of parameters. Further evidence for this picture is that BL Lac objects with intense emission are the most likely sources of UHECRs [6]. The relatively short flares of the CR sources are also consistent with the formation of clusters of particles by several sources in the model with the third set of parameters.

In addition, the CR emission may be affected by the content of protons (nuclei) in the jet plasma or in the region of the accretion disk where the particles are accelerated. According to $[27,28]$, the fraction of the protons in a jet can be $\sim 0.01-0.1$. A change in the proton fraction in the particle acceleration region by several factors will lead to a change in the CR intensity also by several factors, and this will appear as the turn-on and turn-off of the UHECR source.

The turn-on and turn-off of the sources may be the reason why the AGASA array has detected only doublets and one triplet and no clusters with a larger number of particles over 10 years of its operation.

Let us now list the predictions of the model [10] in which the particles are emitted by BL Lac objects with a maximum energy of $10^{27} \mathrm{eV}$. For $L_{B L} \approx 6 \times 10^{45} \mathrm{erg} \mathrm{s}^{-1}$, the CR fluxes in it are too low: arrays with areas $S \sim 100$ and $1000 \mathrm{~km}^{2}$ will detect no more than $10^{-7}$ and $10^{-6}$ particles per year, respectively, from the cluster region. If the power of the directed beam is $L_{B L} \approx 6 \times 10^{45} \mathrm{erg} \mathrm{s}^{-1}$, then the predicted fluxes are too high. Thus, for example, an array with $S \sim 10 \mathrm{~km}^{2}$ will detect $\sim 10^{6}$ particles per year from a single source, in conflict with the measurements. The model with such parameters does not describe the UHECR spectra measured on different arrays [13] either.

It follows from this comparison that $\mathrm{CR}$ data can be a test for some of the theoretical estimates pertaining to AGNs.

\section{Conclusions}

The model in which the sources of UHECRs are AGNs can explain the origin of the particle clusters detected by AGASA. The clusters of particles arrive from areas of the sky with an enhanced density of these objects (the sizes of such sky areas in equatorial coordinates are $\Delta \alpha<9^{0}, \Delta \delta<9^{0}$ ), but can also be detected from individual sources. No clusters can be detected on arrays with an area $S \sim 10 \mathrm{~km}^{2}$.

If the UHECR sources are Seyfert nuclei, then a doublet of particles from a single Seyfert nucleus can be detected on an array with an area $S \approx 100 \mathrm{~km}^{2}$ in an observing time of $T>10$ yr. On an array with $S \sim 3000 \mathrm{~km}^{2}$, a cluster can be detected in a year of its operation.

If UHECRs are emitted by BL Lac objects, then a cluster of particles can be produced by a single source with an emission power in the CR beam of $\sim 10^{33} \mathrm{erg} \mathrm{s}^{-1}$. Doublets and triplets of particles from such single sources can be detected by an array with an area $S \approx 100 \mathrm{~km}^{2}$ in $\sim 2-4 \mathrm{yr}$. If the CR emission power is $\sim 10^{31} \mathrm{erg} \mathrm{s}^{-1}$, then the clusters of particles are emitted by a group of sources. Doublets and triplets of particles will be detected by an array with an area $S \approx 100 \mathrm{~km}^{2}$ also in $\sim 2-4$ yr of its operation.

The CR emission and the cluster formation can be a.ected by the following factors. First, CRs are accelerated in the source quasi-periodically, with a period of 4 to $\sim 25 \mathrm{yr}$ with a relatively short duration of the active state, which is possible, as suggested by the results [26]. Second, the UHECR particle emission may be affected by the changing (from $\sim 0.01$ to 0.1 ) fraction of the protons (nuclei) in the jet plasma or in the particle acceleration region. (Such a change in the proton fraction is consistent with the results [28].)

The variable activity of the sources as well as the decrease and increase in the proton fraction in a jet appears as the turn-on and turn-off of the source. This may be the reason why the AGASA array detected only doublets and one triplet and no clusters with a larger 
number of particles over 10 years of its operation.

The estimates of the CR detection rate from which these conclusions were drawn have been obtained without allowance for the actual time during which the array detected the emission from the sources. In addition, we disregarded the selection of showers by the polar angle of their arrival, that is why no more than half of the emission from the source may be detected. Therefore, a further study of clusters requires taking into account the actual time of CR detection and selecting showers by the polar angle of their arrival.

A comparison of the $\mathrm{CR}$ data with theoretical estimates can serve as a test for the models of AGNs, the UHECR sources.

\section{Acknowledgments}

I am grateful to A.V. Zasov and V.V. Lidskii for discussions and to the referees for remarks.

\section{References}

[1] V.S. Berezinsky et al., Astrophysics of Cosmic Rays. Ed. V.L Ginzburg. Moscow, "Nauka", 1990.

[2] A.V. Uryson, JETP Lett. 64, 77 (1996).

[3] A.V. Uryson, Astron. Zh. 78, 686 (2001) [Astron. Rep. 45, 591 (2001)].

[4] A.V. Uryson, Astron. Astrophys. Trans. 23, 43 (2004).

[5] P.G. Tinyakov and I.I. Tkachev, JETP. Lett. 74, 445 (2001).

[6] D.S. Gorbunov et. al., Astrophys. J. 577, L93 (2002).

[7] P.P. Kronberg, Rep. Progr. Phys. 57, 325 (1994).

[8] A.V. Uryson, Astron. Lett. 27, 775 (2001).

[9] K. Dolag et al., JETP Lett. 79, 583 (2004).

[10] N.S. Kardashev, Mon. Not. R. Astron. Soc. 276, 515 (1995).

[11] A.A. Shatskii and N.S. Kardashev, Astron. Zh. 79, 708, 2002.

[12] A.V. Uryson, Astron. Zh. 81, 99 (2004) [Astron. Rep. 48, 81 (2004)].

[13] A.V. Uryson, Astron. Lett. 30, 816 (2004).

[14] M. Nagano and A.A. Watson, Rev. Mod. Phys. 72, 689 (2000).

[15] E.V. Derishev et al.,Phys. Rev. D68, 043003 (2003).

[16] M.-P. Veron-Cetty and P. Veron, http://www.obshp. fr, 2003.

[17] C. A. Haswell et al.,Astrophys. J. 401, 495 (1992).

[18] M.-P. Veron-Cetty and P. Veron, Astron. Astrophys. 374, 92 (2001).

[19] F. A. Aharonian et al.,Phys. Rev. D66, 023005 (2002).

[20] M. V. Medvedev, Phys. Rev. E67, 045401 (2003).

[21] K. Greisen, Phys. Rev. Lett. 16, 748 (1966).

[22] G. T. Zatsepin and V.A. Kuzmin, JETP Lett.4, 78 (1966).

[23] N. Hayashida et al., astroph/0008102, 2000.

[24] B. N. Afanasiev et al., Proceedings of International Symposium

"Extremely High Energy Cosmic Rays: Astrophysics and Future Observatories".

Ed. M. Nagano. Inst. Cosmic-Ray Res., Tokyo, 1996.

[25] V. A. Lipovetskii et al., Communications of the SAO. No 55, 1987.

[26] T. B. Pyatunina et al., astroph/0502173, 2005.

[27] S. A. Koryagin, Private communication. 2004.

[28] V. V. Zheleznyakov and S. A. Koryagin, Astron. Lett. 28, 809 (2002). 